\title{
Research on Welding Machine Control System
}

\author{
Jiandong Yang ${ }^{1, a}$, Xin $\mathrm{Qi}^{2, \mathrm{~b}}$ \\ ${ }^{1}$ Jilin Business and Technology College,Changchun,130062,China \\ ${ }^{2}$ Mechanical Manufacture and Automation ,Changchun University of Science and \\ Technology, Changchun,130022, China

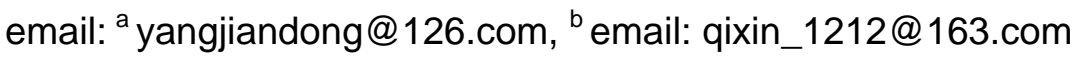

Keywords: Welding; SCM; PLC; IPC; Motion Control Card

\begin{abstract}
The characteristics and applications of the system for welding machines based on SCM , based on PLC control system and PC-based industrial control system was introduced. It has certain significance to the design of welding machine control system.
\end{abstract}

\section{Introduction}

With the rapid development of modern network technology in this society, the welding technology management has been transformed from the previous manual operation to the network management. The technologies of applied computing and telecommunications and other technologies have achieved the functions of off-line programming, remote monitoring, diagnostics and maintenance, so that the welding machine automatic control process and production management develop toward integration. Using the advanced sensor technology, computer technology and intelligent control technology of welding automation technologies can easily cope with the various complicated environment and the changing welding conditions. Welding automation technology means the comprehensive technology that combining the automatic intelligent control system, the welding jig, clamping and locating system, the digital welding equipment and high-quality welding materials, to reduce the cost of mass production, to ensure the high quality and efficiency of production.

With the continuous development and updating of numerical control system for CNC system, the system has been developed from the CNC system adapted electron tube to the CNC system based on industrial PC machine. The CNC system applied on welding machine is also under developing and the following kinds of welding machine control systems have appeared ${ }^{[1]}$.

\section{CNC system Based on SCM}

The single chip has been widely used in the spot welding control system because of the advantages of light mass, small volume, cheap price and reliable performance. The spot welding controllers based on SCM designed by Chen Yiping from Nanchang Aeronautical University have achieved precise control of electric current and maintain fast response; the TIM4002 in the American Square company and the BOSCHE81 in West Germany BOSCH company are all based on SCM control welding equipment ${ }^{[2]}$.

SCM is usually used for storage, call, parameter adjustment and timing control in spot welding process, while also generally be used for single-parameter control. Only using single chip microcomputer is unable to complete the complex calculation of intelligent control .Software design of single-chip system adapts assembly language programming which is complex and error-prone. Its development cycle is longer and usually only applied to one-on-one products and services. Its compatibility, commonality and expansibility is worse than other control systems.

Though the CNC welding machine adopting SCM costs low but has poor openness, limited hardware and software resources and the machine operation is not convenient, man-machine interface is not friendly, therefore, it generally used to develop simple and economical CNC welding machine. 


\section{CNC system based on programmable logic controller (PLC)}

PLC has the advantages of high reliability, strong anti-jamming capability, broad applicability, easy to be learned and used, short system design period, easy to be maintained, easy to be transformed, small size, light weight and low energy consumption, so the control unit adopting PLC can make the system link simple, reduce the number of contactor control links and greatly reduce the failure rate. Using the PLC as the core welding machine control system, on the one hand, can control the welding machine, on the other hand, communicates with the man-machine interface.

PLC is adapted as the lower computer in NC welding machine to directly interact with the hardware[3], and the touch screen is used to display in order to satisfy the complex requirements of the welding process and friendly human-machine interface, thus develop the welding machine CNC system based on PLC.The system chart of a welding machine control system based on PLC is shown in Fig.1.

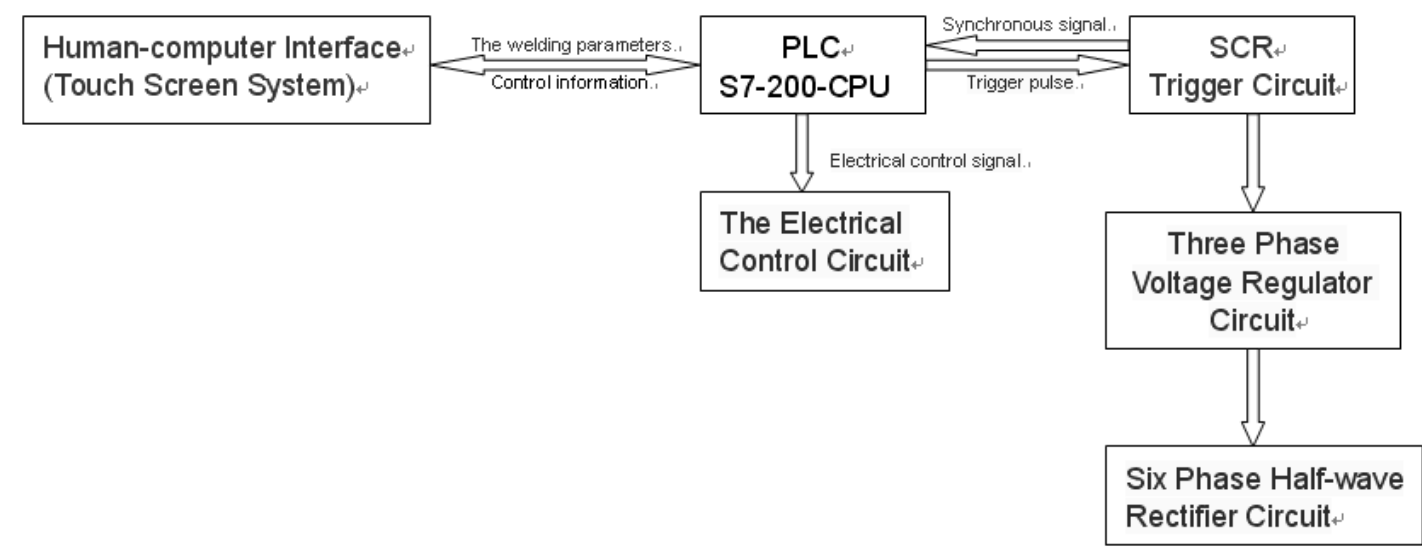

Fig.1. Welding machine control system based on PLC

\section{CNC system based on industrial control computer (industrial PC)}

CNC system based on industrial PC is a new and open CNC system, and is used more and more widely in machine tool manufacturing industry. It not only has the common characteristics with the universal computer, but has open modular structure, strong data processing ability, good practicability and industrial environment adaptability. However, the PC machine is difficult to real-time control, especially in the high real-time requirement of control system[4]. Therefore, the control device can adopt the form of "PC+ motion control card" to compose the NC system. This form is becoming more and more with the popularization of PC machines, and this will be a main trend of motion control system. It can make full use of the computer resource and can be used for the equipment which has complex motion process and trajectories, and requires high flexibility.

The PC machines are used to handle non-real-time part and motion control card is used to real-time part. PC machine as the host machine is used to realize the man-machine interface, complete the input of processing program, pre compiled, calculate the trajectory and speed, simulate the graphics of processing parts. The motion control card is used as the lower computer to deal with some real-time machine signal. In the welding machine numerical control systems, a PC or industrial PC mother board is used as the CNC platform, and insert the PC/ISA slot on the main board (or mother) to achieve various modules of NC functions, such as motion control card, DA board, I/O board, thus a highly flexible CNC system is constituted. Make full use of the resources of the Windows operating system and the advanced language programming can make the CNC software system has more powerful functions.

This control system has simple structure, and there is no need to extend complex peripheral circuit. The cycle of software design is short, and this system can be debugged and controlled conveniently, 
it also has high reliability and the expansion of function is convenient. It can make full use of rich resources on PC machine to improve the processing ability of the system effectively. The control system hardware structure is shown as Fig.2.

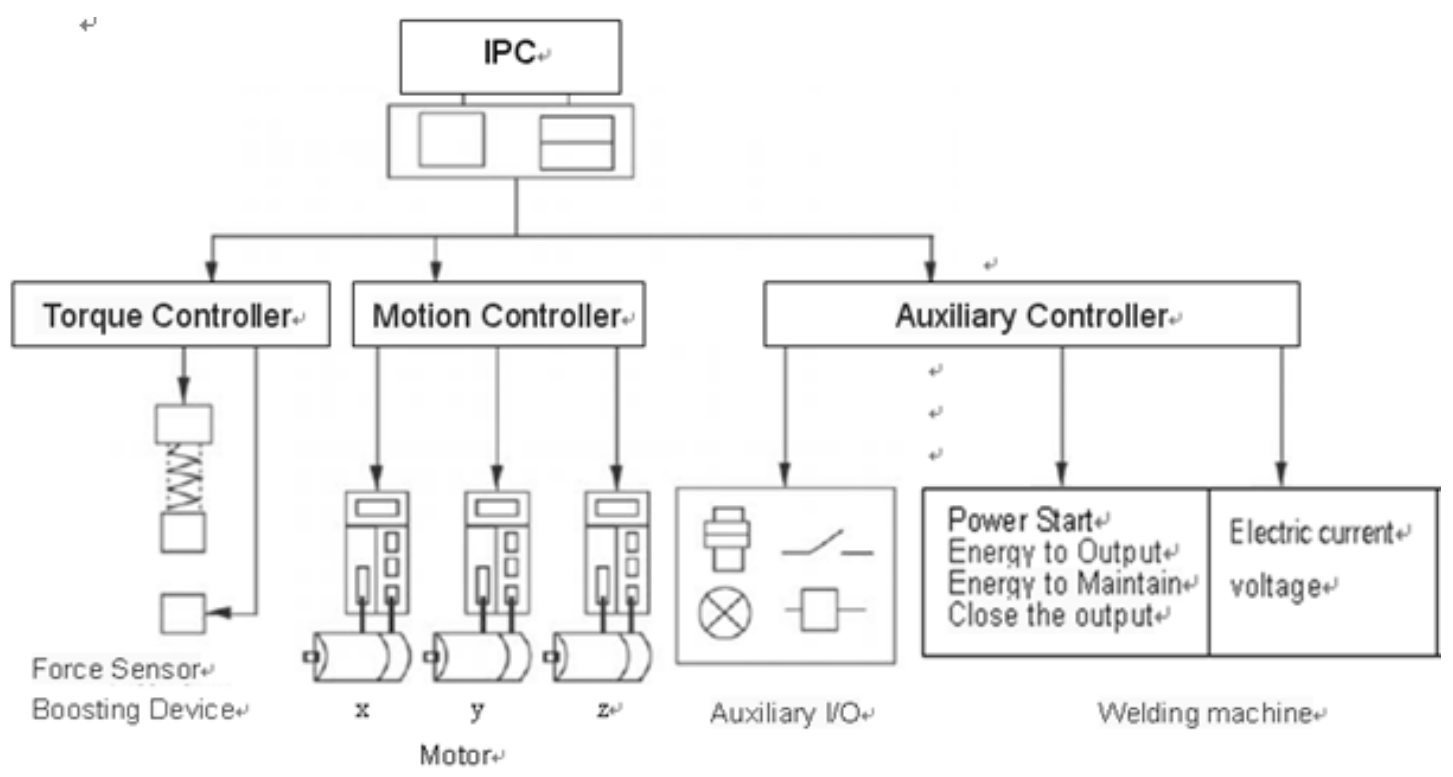

Fig.2. The control system hardware structure

In addition, the welding CNC system based on the host computer(IPC) and the lower computer (PLC) has been used in the actual production. The host computer(IPC) is used for dynamic display and parameter setting, the lower position machine (PLC) is used to control the welding equipment to complete the welding process.

\section{Conclusion}

This paper has introduced three common control systems in the current NC welding machines: (1)CNC system based on SCM. (2)CNC system based on industrial control computer (industrial PC) . (3) CNC system based on programmable logic controller (PLC). Contrasting and analyzing the advantages and disadvantages of each control system form and the application situations can provide useful information for the foundation and reference for choosing the suitable control system when designing a welding machine.

\section{Acknowledgements}

The authors gratefully acknowledge the funding of this study by Science and Technology Development Plan of Jilin province (20130204024GX) and Talent Development Project of Jilin province (20091328) and Venture Fund Project for Personnel Studying Abroad of Jilin province (2010273).

\section{References}

[1] Wang Jianbing. Exploitation and Application Research of NC welding machine[D].HeFei: HeFei University of Technology,2006

[2] Ling Min. Research on the spot welding control system based on PLC[D].NanChang: Nanchang Hangkong University,2012

[3] Li Yongjiu.Welding Teaching Robot Reach.[D].WuHan.HuBei: Huazhong University of Science and Technology.2008 
[4] Huang Zhengyan. Research and development of plane type arc welding robot [D].TianJin:TianJin University.2008

[5] Jiandong Yang,Hui Yan,Chunlin Tian,Jing Li ,Exploration on Automatic Welding Methods for Battery Pack of New Energy Automobiles[J]. AMR(ERPE2014),2014(5):923-926. 